\title{
Graceful $V^{*} 2 F_{n}$-tree
}

\author{
D. R. Kirubaharan ${ }^{1}$, Dr. G. Nirmala ${ }^{2}$ \\ ${ }^{1}$ Research Scholar, Manonmaniam Sundaranar University, Tirunelveli \&Asst Professor in Mathematics, \\ A.V.V.M. Sri Pushpam College, Poondi, Thanjavur, Tamilnadu. India, \\ ${ }^{2}$ Research Advisor, Kunthavai Nachiyar Govt Arts College, Thanjavur, Tamilnadu. India
}

Abstract: The concept of graceful labeling was introduced by Solomon Wolf Golomb (May 30, 1932). In this paper we discuss graph and its graceful labeling. Finally we show that $V^{*} 2 F_{n}$ is graceful for an $n$.

Keywords: Graceful tree, graceful labeling, root, stem, branch, leaf, flower.

\section{Introduction}

The last two decades have witnessed an upsurge of interest and activity in graph theory, particularly among applied mathematicians and engineers. The past 30 years has been a period of intense activity in graph theory both pure and applied. A great deal of research has been done and is being done in this area.

Graph theory is one of the most flourishing branches of mathematics with wide application to combinational problems. Graphs are usually represented by diagrams using a point for each vertex and a line for each edge. A graph $G$ is an ordered triple of $(V(G), E(G), \psi)$. If $e$ is an edge and $\psi(e)=(u, v)$, then we say that $e$ is an edge joining $u$ and $v$ and the vertices $u$ and $v$ are called the ends of $e$. Now label each edge with the absolute difference of the endpoints of the concerned edge. The labeling is graceful, if the edges are labeled $1,2, \ldots, n$ inclusive.

A graceful labeling on a graph with $p$ vertices and $q$ edges is a one to one map taking the vertices into the integers $0,1, \ldots q$ with the property that each edge $u v$ is assigned by the label $|f(u)-f(v)|$.

A graceful graph is a graph that has atleast one graceful labeling.

\subsection{Definition}

A walk is a list $v_{0}, e_{1}, v_{l}, e_{2}, \ldots, e_{k}, v_{k}$ of vertices and edges such that, for $1 \leq i \leq k$, the edge $e_{i}$ has end points $v_{i-l}$ and $v_{i}$. A trail is a walk with no repeated edge. A $u, v$-walk or $u, v$-trail has first vertex $u$ and last vertex $v$; that is $u$. $v$ are its endpoints. A $u, v$-path is a path whose vertices of degree 1 (its endpoints are $u$ and $v$ ); the other vertices are internal vertices and of degree 2 . The length of a walk, trail, path, or cycle is its number of edges. A walk or trail is closed if its endpoints are the same.

\subsection{Example}

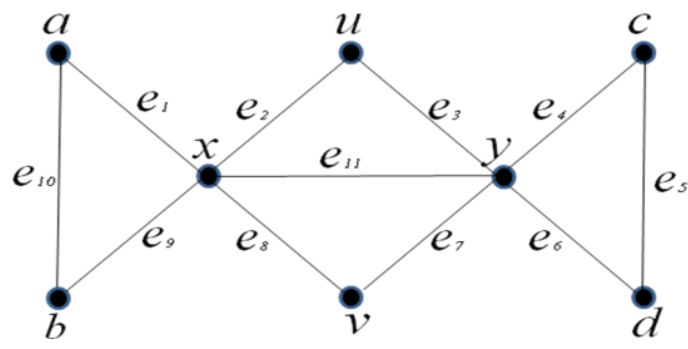

Fig.1: Path graph

In the above graph $a, e_{1}, x, e_{2}, u, e_{3}, y, e_{4}, c, e_{5}, d, e_{6}, y, e_{7}, v, e_{8}, x, e_{9}, b, e_{10}, a$ specifies a closed walk of length 10. Omitting the first two steps yields a closed trail and it has five cycle $(a, b, x),(c, y, d),(u, x, y),(x, y, v),(u, x, v, y)$

\subsection{Definition}

A graph $G$ is connected if there is a path between every pair of vertices. 


\subsection{Definition}

A graph with no cycle is acyclic. Collection of trees is called forest. A tree is a connected acyclic graph. A leaf in a tree (or pendent vertex) is a vertex of degree 1.

\subsection{Definition}

If in a tree there is one vertex which is distinguished from all other vertices then the vertex is called root and the tree is called a rooted tree and also the root is denoted by $r$.

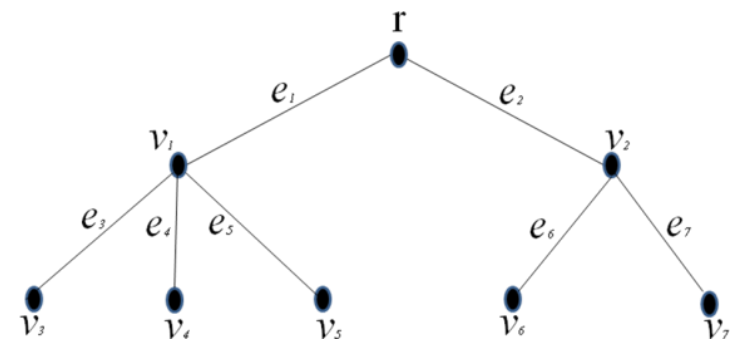

Fig.2: Rooted tree

\subsection{Definition}

A binary tree is a rooted tree where each vertex has atmost two children.

\subsection{Definition}

A star is a special kind of tree consisting of one vertex adjacent to all the other vertices and a star is denoted by $K_{l, n-l}$.

\subsection{Definition}

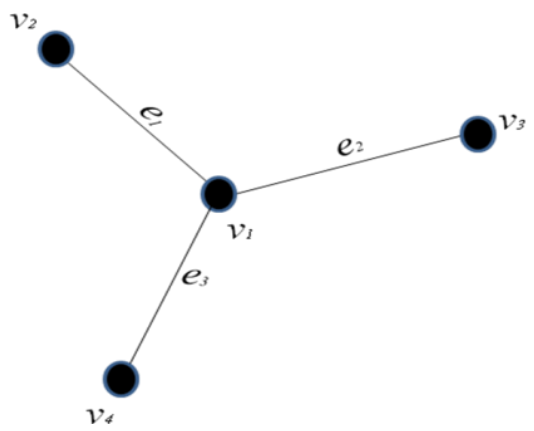

Fig.3: $K_{l, 3}-$ Star graph

A matching in a graph is a subset of edges in which no two edges are adjacent. The vertices incident to the edges of a matching $M$ are saturated by $M$; the others are unsaturated (we say $M$-saturated and $M$ unsaturated vertices)

\subsection{Definition}

A perfect matching in a graph is a matching that saturates every vertex of a graph $G$.

\subsection{Definition}

Given a matching $M$, an $M$-alternating path is a path that alternates between edges in $M$ and edges not in $M$. An $M$-alternating path whose endpoints are unsaturated by $M$ is an $M$-augmenting.

\subsection{Definition}

Let $M$ be a matching in a graph $G$, and let $u$ be an $M$-unsaturated vertex. A flower is the union of two $M$-alternating paths from $u$ that reach a vertex $x$ on steps of opposite parity. The stem of the flower is the maximal common initial path (of non negative even length).The blossom of the flower is the odd cycle obtained by deleting the stem.

\subsection{Theorem (Berge)}

A matching $M$ in $G$ has maximum size if and only if $G$ has no $M$-augmenting Path. 


\subsection{Example}

In the graph below, the matching $M$ indicated in bold, a search for shortest $M$-augmenting paths from $u$ reaches $x$ via the unsaturated edge $a x$. If we do not also consider a longer path reaching $x$ via a saturated edge, then we miss the augmenting path $u, e_{1}, v, e_{2}, a, e_{3}, b, e_{4}, c, e_{5}, d, e_{6}, e, e_{7}, f, e_{8}, g, e_{9}, h, e_{10}, i, e_{11}, j, e_{12}, x, e_{14}, y$.

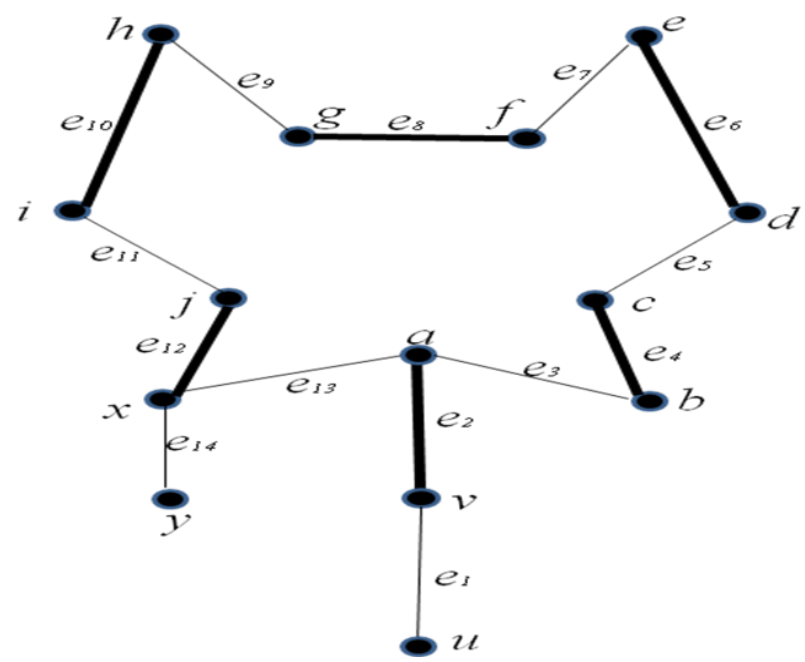

Fig.4: Flower graph

If an exploration of $M$-alternating paths from $u$ reaches a vertex $x$ by an unsaturated edge in one path and by a saturated edge in another path, then $x$ belongs to an odd cycle. Alternating paths from $u$ can diverge only when the next edge is unsaturated; when the next edge is saturated there is only one choice for it.

$P_{1}$ and $P_{2}$ are different paths from $u$ reaches $x$ and its union makes a flower.

$$
\begin{aligned}
P_{1} & =\left\{u, e_{1}, v, e_{2}, a, e_{3}, b, e_{4}, c, e_{5}, d, e_{6}, e, e_{7}, f, e_{8}, g, e_{9}, h, e_{10}, i, e_{11}, j, e_{12}, x\right\} \\
P_{2} & =\left\{u . e_{1}, v, e_{2}, a, e_{13}, x\right\} \\
P & =P_{1} \mathrm{U} P_{2} \\
& =\text { flower }
\end{aligned}
$$

\subsection{Definition}

A graceful labeling of a graph $G$ with $m$ edges is a function $f: v(G) \rightarrow\{0, \ldots . m\}$ such that, distinct vertices receive distinct numbers and $\{|f(x)-f(y)|: x y \in E(G)\}=\{1, \ldots m\}$ and a graph is graceful, if it has a graceful labeling.

\subsection{Definition}

A rooted tree (Bi-tree) is a V-tree if it has only two branches which are leaves.

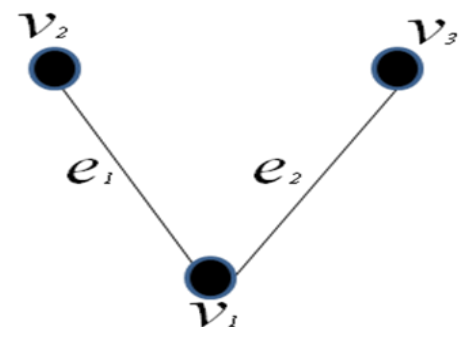

Fig.5 : V-tree

\subsection{Definition}

The $\mathrm{V}^{*} 2 \mathrm{~F}_{n}$ is a V-tree, it combined with $n$-stars in each of the two branches. 


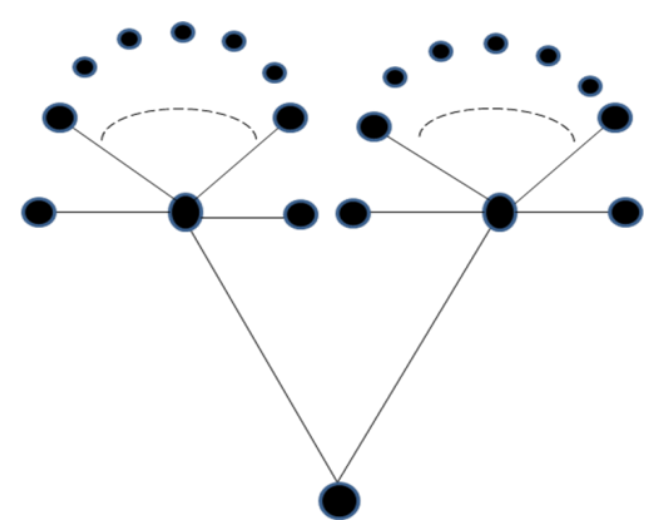

Fig. 6(a): $\mathrm{V} * 2 \mathrm{~F}_{n}$

\subsection{Theorem}

The tree $\mathrm{V}^{*} 2 \mathrm{~F}_{n}$ is graceful.

\section{Proof:} as bellow.

Let the tree $\mathrm{V}^{*} 2 \mathrm{~F}_{n}$ be obtained by merging $n$ copies of stars $\mathrm{F}_{n}$ with $\mathrm{V}$ - tree, which is given

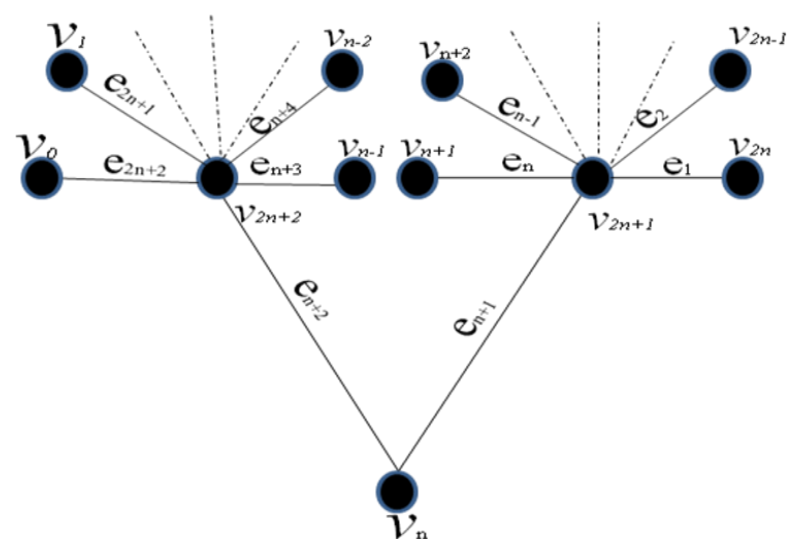

Fig. 6(b) : Graceful labeling $\mathrm{V} * 2 \mathrm{~F}_{n}$

Let $p=2 n+3$ be the number of vertices and $q=2 n+2$ be the number of edges in the $\mathrm{V}^{*} 2 \mathrm{~F}_{n}$ - tree. Now, define a labeling $f$ on the vertex set of $\mathrm{V}^{*} 2 \mathrm{~F}_{n}$ by $f\left(v_{i}\right)=i$ such that $i=0$ to $2 n+2$.

The edge set labeling $e$ is defined by $e(u v)=|f(u)-f(v)|$, for any edge $u v$ in the tree $\mathrm{V}^{*} 2 \mathrm{~F}_{n}$. Thus the given tree with the vertex labeling $f$ and edge labeling $e$ becomes a graceful tree. Hence $\mathrm{V}^{*} 2 \mathrm{~F}_{n}$ is a graceful tree.

\subsection{Example}

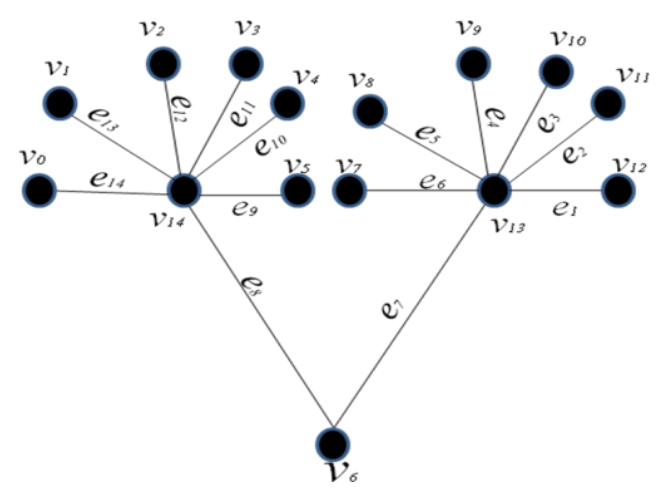

Fig.6(c) : Graceful labeling $\mathrm{V}^{*} 2 \mathrm{~F}_{6}$ 
Let $p=15, q=14$ be the number of vertices and edges respectively and $n=6$ be the number of stars merges in each branch of the V-tree. Define a vertex labeling ' $f$ ' on the $\mathrm{V}^{*} 2 \mathrm{~F}_{6}$ by $f\left(v_{i}\right)=i$, $i=0$ to 14 and edge labeling by $e(u v)=|f(u)-f(v)|$ for any edge $u v$ in the tree, $\mathrm{V}^{*} 2 \mathrm{~F}_{6}$.

\subsection{Theorem:}

\section{Proof:}

$\mathrm{V}^{*} 2 \mathrm{~F}_{n}$ is graceful if $n$ is even. as bellow

Let the tree $\mathrm{V}^{*} 2 \mathrm{~F}_{n}$ be obtained by merging $n$ copies of stars $\mathrm{F}_{n}$ with $\mathrm{V}$ - tree, which is given

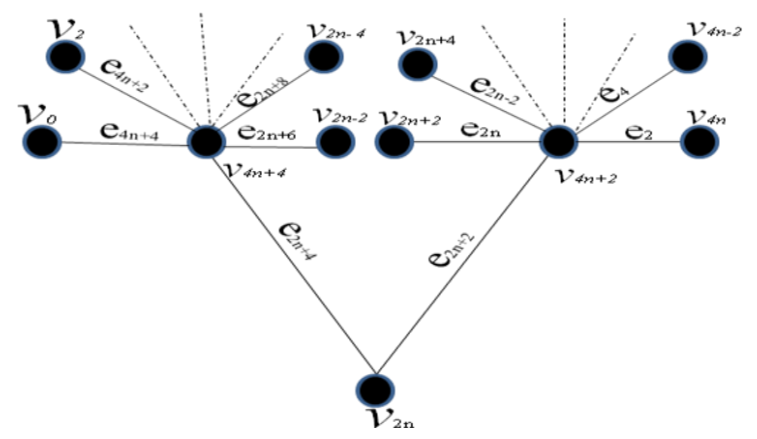

Fig.7(a) : Even graceful labeling $\mathrm{V}^{*} 2 \mathrm{~F}_{n}$

Let $p=4 n+5$ be the number of vertices labeling by even integers and $q=4 n+4$ be the number of edges labeling by even integers in the $\mathrm{V}^{*} 2 \mathrm{~F}_{n}$ - tree. Now, define a labeling $f$ on the vertex set of $\mathrm{V}^{*} 2 \mathrm{~F}_{n}$ by $f\left(v_{i}\right)=2 i$ such that $i=0$ to $4 n+4$

$\rightarrow(2)$

The edge set labeling $e$ is defined by $e(u v)=|f(u)-f(v)|$, for any edge $u v$ in the tree $\mathrm{V}^{*} 2 \mathrm{~F}_{n}$. Thus the given tree with the vertex labeling $f$ and edge labeling $e$ becomes a graceful tree if $\mathrm{n}$ is even. Hence $\mathrm{V}^{*} 2 \mathrm{~F}_{n}$ is a graceful tree.

\subsection{Example}

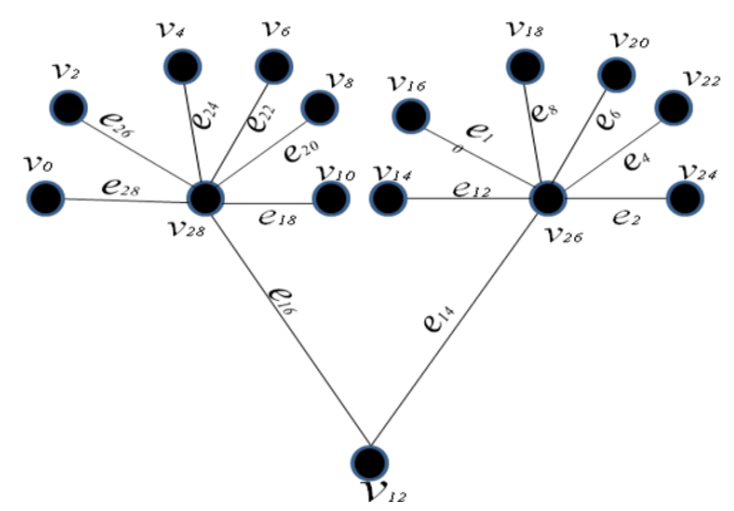

Fig.7(b) : Even graceful labeling $\mathrm{V}^{*} 2 \mathrm{~F}_{6}$

Let $p=15, q=14$ and $p, q$ are having only even number of vertices and edges respectively and $n=6$ be the number of stars merges in each branch of the V-tree. Define a vertex labeling ' $f$ ' on the $\mathrm{V}^{*} 2 \mathrm{~F}_{6}$ by $f\left(v_{i}\right)=2 i, i=0$ to 28 and edge labeling by $e(u v)=|f(u)-f(v)|$ for any edge $u v$ in the tree, $\mathrm{V}^{*} 2 \mathrm{~F}_{6}$.

\subsection{Theorem:}

\section{Proof:}

$\mathrm{V}^{*} 2 \mathrm{~F}_{n}$ is graceful if $n$ is odd. shown as below.

Let the tree $\mathrm{V}^{*} 2 \mathrm{~F}_{n}$ be obtained by merging $n$ copies of stars $\mathrm{F}_{n}$ with $\mathrm{V}$ - tree, which is 


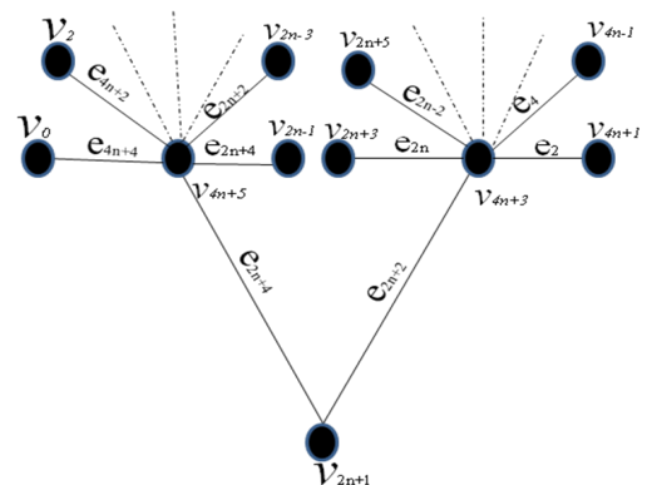

Fig.8(a) : Odd graceful labeling $\mathrm{V}^{*} 2 \mathrm{~F}_{n}$

Let $p$ be the number of vertices with odd integers of labeling and $q$ be the number of edges with even number of labeling in $\mathrm{V}^{*} 2 \mathrm{~F}_{n}$ - tree. According the definition of graceful labeling $f$ on the vertex set of $\mathrm{V}^{*} 2 \mathrm{~F}_{n}$ by $f\left(v_{i}\right)=2 i+1$ such that $i=0$ to $4 n+4$

The edge set labeling $e$ is defined by $e(u v)=|f(u)-f(v)|$, for any edge $u v$ in the tree $\mathrm{V}^{*} 2 \mathrm{~F}_{n}$. Thus the given tree with the vertex labeling $f$ and edge labeling $e$ becomes a graceful tree if $n$ is odd. Hence $\mathrm{V}^{*} 2 \mathrm{~F}_{n}$ is a graceful tree.

\subsection{Example}

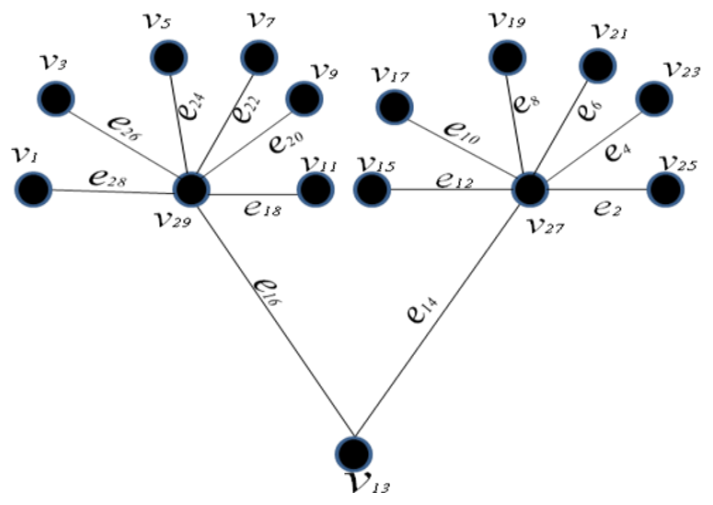

Fig.8(b) : Odd graceful labeling $\mathrm{V}^{*} 2 \mathrm{~F}_{6}$

Let $p$ and $q$ be the number of vertices and edges. The vertices are labeling by odd integers and the edges are labeling by even numbers respectively. Because the difference between two odd integers is even and if $n=6$ be the number of stars in each branch of the V-tree. Define a vertex labeling ' $f$ ' on the $\mathrm{V}^{*} 2 \mathrm{~F}_{6}$ by $f\left(v_{i}\right)=2 i+1, \quad i=0$ to 28 and edge labeling by $e(u v)=|f(u)-f(v)|$ for any edge $u v$ in the tree, $\mathrm{V}^{*} 2 \mathrm{~F}_{6}$ is graceful tree.

\section{Conclusion}

In this paper we have proved that $\mathrm{V} * 2 \mathrm{~F}_{\mathrm{n}}$ is graceful when $n$ is odd or even. So we conclude that $\mathrm{V} * 2 \mathrm{~F}_{n}$ is graceful for an $n$.

\section{Reference}

[1] Douglas B. West., Introduction to Graph Theory, Second edition, Dorling Kindersley (India) Pvt. Ltd. (2001).

[2] Edmonds. J., Paths, trees, and flowers, Can.J.Math.17(1965),449-467.

[3] Frank Harary., Graph Theory, Narosa Publishing House, 1969.

[4] Golomb. S.W., How to number a graph in Graph Theory and computing (ed. R.C. Road) 1, Academic Press, 1972.

[5] Graham. R.L and Sloane. N.J.A.,An Additive Bases and Harmonious Graph, SIAMJ. Alg. Discrete. Math., 1 (1980) 382 - 404

[6] Harary. F., Graph Theory, Addison Wesley, Reading Massachusetts, 1969.

[7] Murugan. M., Topics in Graph Theory and Algorithms.

[8] Rosa. A.,On certain vertex valuation of the vertices of a graph. In Theory of Graphs, Intl. Sump. Rome 1966, Gordon and Breach, Dunod, (1966), 349-355.

[9] Kirubaharan.D.R.,Arunraj.,Application of gracefulness on v-tree,vol.6,No.2.2012,104-105 